\author{
MITSUBISHI ELECTRIC RESEARCH LABORATORIES \\ http://www.merl.com
}

\title{
Saturation-robust SAR Image Formation
}

\author{
Wei, D.; Boufounos, P.T.
}

TR2011-015 May 2011

\begin{abstract}
The formation of synthetic aperture radar (SAR) images is formulated as an inverse problem, a flexible approach suitable for a variety of acquisition systems and signal models. This paper focuses on increasing robustness to data saturation, specifically by optimizing a one-sided quadratic cost function to promote consistency with the received data. We model the SAR acquisition process using a linear function and we present an efficient implementation of this function and its adjoint for use in iterative optimization algorithms. Improved image quality and robustness to saturation are observed in experiments on synthetic images. Preliminary work on controlling azimuth ambiguities and incorporating image models enables saturation-robust reconstruction from satellite SAR data as well.
\end{abstract}

IEEE International Conference on Acoustics, Speech and Signal Processing (ICASSP)

\footnotetext{
This work may not be copied or reproduced in whole or in part for any commercial purpose. Permission to copy in whole or in part without payment of fee is granted for nonprofit educational and research purposes provided that all such whole or partial copies include the following: a notice that such copying is by permission of Mitsubishi Electric Research Laboratories, Inc.; an acknowledgment of the authors and individual contributions to the work; and all applicable portions of the copyright notice. Copying, reproduction, or republishing for any other purpose shall require a license with payment of fee to Mitsubishi Electric Research Laboratories, Inc. All rights reserved.
} 



\section{SATURATION-ROBUST SAR IMAGE FORMATION}

\section{Dennis Wei}

\author{
Massachusetts Institute of Technology \\ Digital Signal Processing Group \\ Cambridge, MA 02139 \\ dwei@mit.edu
}

\author{
Petros T. Boufounos \\ Mitsubishi Electric Research Laboratories \\ Cambridge, MA 02139 \\ petrosb@merl.com
}

\begin{abstract}
The formation of synthetic aperture radar (SAR) images is formulated as an inverse problem, a flexible approach suitable for a variety of acquisition systems and signal models. This paper focuses on increasing robustness to data saturation, specifically by optimizing a one-sided quadratic cost function to promote consistency with the received data. We model the SAR acquisition process using a linear function and we present an efficient implementation of this function and its adjoint for use in iterative optimization algorithms. Improved image quality and robustness to saturation are observed in experiments on synthetic images. Preliminary work on controlling azimuth ambiguities and incorporating image models enables saturation-robust reconstruction from satellite SAR data as well.
\end{abstract}

Index Terms - Synthetic aperture radar, inverse problems, data saturation

\section{INTRODUCTION}

The study of inverse problems is a well-established discipline with several applications in a variety of fields, including signal processing. The theory of inverse problems examines when and how functions can be inverted so as to infer the input to the function from the output. Such problems are abundant in signal acquisition and processing systems and the formulation of these problems as inverse problems has produced significant results in the areas of de-noising, de-blurring and super-resolution.

With appropriate modeling of an acquisition system, reconstruction becomes an inverse problem. Specifically, the signal of interest is processed by the function implemented by the acquisition system, and the reconstruction is the solution to the corresponding inverse problem. With such modeling, the uniqueness of the solution or, if the solution is not unique, the accuracy of the solution is of significant interest.

In this paper, we study the formation of synthetic aperture radar (SAR) images as an inverse problem. SAR is widely used in remote sensing to obtain high-resolution, wide-area radar images using a relatively small antenna. High resolution is made possible by exploiting the motion of the platform carrying the antenna (usually a plane or satellite) to synthesize a much larger aperture. The acquisition process in SAR involves transmitting pulses at regular intervals as the platform moves and recording the reflections to gain information about the reflectivity of the surface below. We formulate a general optimization approach to the formation of a reflectivity image from these measurements. While the results we present focus on reconstructing images from saturated measurements, they are straightforward to extend to a wide range of scenarios such as coarse quantization or missing data.

In Section 2, we provide some background on inverse problems and discuss SAR image formation and data saturation in this context. In Section 3, we describe key aspects of our algorithm for saturationrobust SAR image formation. Experimental results on synthetic and satellite SAR data are reported in Section 4.

\section{BACKGROUND}

\subsection{Inverse Problems}

A common inverse problem in signal processing is the recovery of a signal $\mathbf{x}$ from a set of measurements $\mathbf{y}$,

$$
\mathbf{y}=A(\mathbf{x})+\mathbf{n}
$$

where the function $A$ models the acquisition system and $\mathbf{n}$ represents measurement noise. The goal of the reconstruction process is to determine a signal estimate $\widehat{\mathbf{x}}$ that is close to $\mathbf{x}$.

If the acquisition function $A$ is easily invertible and the measurement noise is negligible, an obvious choice is to use the inverse of $A$ to determine x. However, this method may fail if $A$ is not injective, i.e., the data can be explained by multiple signals, or if there is considerable noise. A more general approach is to estimate $\mathbf{x}$ by the following minimization:

$$
\widehat{\mathbf{x}}=\arg \min _{\mathbf{x}} f(\mathbf{y}, A(\mathbf{x}))+\lambda g(\mathbf{x}),
$$

where $f(\cdot, \cdot)$ is a cost function measuring data fidelity according to the properties of the acquisition system and the noise, $g(\mathbf{x})$ is a regularizer that incorporates knowledge about the signal of interest and penalizes unwanted solutions, and $\lambda$ controls the trade-off between the two terms. The formulation in (2) offers flexibility in accommodating a range of acquisition scenarios and signal models.

In the special case where $f(\mathbf{y}, A(\mathbf{x}))=\|\mathbf{y}-A(\mathbf{x})\|_{2}, g(\mathbf{x})=$ $0, A$ is linear, and the system is overdetermined, the solution to (2) is to use the pseudoinverse $A^{\dagger}$. The pseudoinverse is also the solution if instead the system is underdetermined and $g(\mathbf{x})=\|\mathbf{x}\|_{2}$, i.e., a least-energy solution is desired.

\subsection{SAR Image Formation}

The formation of SAR images can be formulated as an inverse problem. Specifically, the ideal SAR acquisition process can be viewed as a linear system, i.e., an instance of (1) in which $\mathrm{x}$ represents a 2-D image of surface reflectivity, $\mathbf{y}$ represents the received data, and the function $A$ is linear. We will henceforth use the matrix notation $\mathbf{A}$ to 
represent a linear acquisition function. The received data are usually arranged in a 2-D form similar to the SAR image. The samples of each reflected pulse can be thought of as forming a row vector, with different reflected pulses stacked together to form a matrix of data. Each row of the matrix corresponds to a different position of the platform along its path. The dimension along the path is referred to as the azimuth. Each column corresponds to a delay from the transmission of a pulse. Since the delay is proportional to the distance the pulse has traveled, this second dimension is referred to as the range.

Several existing image formation algorithms can be interpreted from the viewpoint of inverse problems as computing approximations to the pseudoinverse $\mathbf{A}^{\dagger}$. The approximations allow for efficient implementations. For example, many algorithms rely on pulse compression, the approximate deconvolution of a received signal through correlation with the transmitted pulse. The correlation can be made highly efficient by using Fast Fourier Transform (FFT) algorithms. Pulse compression corresponds to the exact inverse (at least within the bandwidth of interest) if the received pulse is sampled at the Nyquist rate, and corresponds closely to the pseudoinverse if the received pulse is slightly oversampled, which is usually the case in practical SAR systems.

One of the most widely used image formation algorithms is the Chirp Scaling Algorithm (CSA), first published in [1] and also described in [2]. A simplified block diagram for the CSA is shown in Fig. 1. In addition to pulse compression, the CSA also employs a technique known as chirp scaling, which approximates a timevarying delay of a linear chirp signal by means of multiplications with two other chirp signals. Chirp scaling is used in the CSA to correct for range migration, the variation in distance to a given target (and hence its associated delay) caused by the motion of the platform. The CSA is a very efficient algorithm due to its composition in terms of FFTs and multiplications only. For this reason, it will form the basis for our method of computing the acquisition function $\mathbf{A}$ and its adjoint as described in Section 3.1.

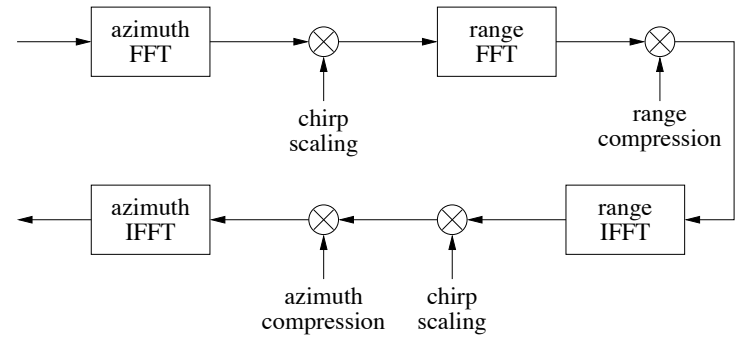

Fig. 1. Block diagram for the CSA.

Saturation of data values as discussed in Section 2.3 is a significant problem in SAR acquisition because of large fluctuations in data amplitudes and the use of low-precision quantizers. Moreover, conventional image formation algorithms do not specifically address saturation. In the next section, we discuss how data saturation can be handled within the inverse problem framework.

\subsection{Saturation and Inverse Problems}

Signal saturation is a very common problem encountered in analogto-digital (A/D) conversion systems. The electronics have a finite voltage range beyond which the signal amplitude is not allowed to vary and the quantizer uses a finite number of quantization levels. If the input signal amplitude varies beyond saturation thresholds $\pm T$, then the acquired amplitude is saturated to $\pm T$.
Although saturation on its own is undesirable, in the presence of severe quantization it can be beneficial. Specifically, increasing the gain of the signal and promoting saturation also increases the signalto-quantization noise ratio in the unsaturated measurements. If the saturation is appropriately taken into account, overall reconstruction performance can be increased [3, 4].

The most common approach to handling saturation-using the saturated values at face value in the reconstruction as if no saturation occurred-produces severe artifacts. Instead, the reconstruction error can be reduced using a consistent reconstruction approach, i.e., ensuring that the reconstructed signal estimate produces the same saturation if re-acquired. Consistent reconstruction, first introduced in [5], significantly improves the reconstruction error when used with quantized and saturated measurements $[6,5,4]$. This is the approach we use in this paper.

Using the same formulation as before, we can represent a linear system with finite dynamic range using

$$
\mathbf{y}=S(\mathbf{A x}),
$$

where $S(\cdot)$ is the non-linear scalar saturation function

$$
S(x)=\operatorname{sign}(x) \min \{|x|, T\}
$$

applied element-wise to all the components of its input.

Instead of the classical least-squares fidelity criterion, to ensure consistent reconstruction we enforce a one-sided quadratic penalty on saturated measurements. More specifically, we use the cost function

$$
\begin{aligned}
f(\mathbf{y}, \mathbf{A x}) & =\frac{1}{2} \sum_{i} r_{i}^{2}\left(y_{i},(\mathbf{A x})_{i}\right), \\
r_{i}\left(y_{i},(\mathbf{A} \mathbf{x})_{i}\right) & = \begin{cases}(\mathbf{A} \mathbf{x})_{i}-y_{i} & \left|y_{i}\right|<T \\
\left((\mathbf{A x})_{i}-T\right)^{-} & y_{i} \geq+T \\
\left((\mathbf{A x})_{i}+T\right)^{+} & y_{i} \leq-T\end{cases}
\end{aligned}
$$

where $(y)^{-}=\min \{y, 0\},(y)^{+}=\max \{y, 0\}$, and $i$ indexes the real and imaginary parts of the data separately. This penalty, commonly used in other consistent reconstruction approaches, has the advantage that it penalizes inconsistent solutions, is simple to optimize and, due to its quadratic nature, provides significant robustness to additive noise before the saturation occurs.

Although minimizing the saturation-robust cost in (5) often produces acceptable solutions, a signal model can also be used to further resolve the ambiguities inherent in saturation and quantization.

\section{SATURATION-ROBUST SAR IMAGE FORMATION}

\subsection{Implementation of $\mathbf{A}$ and $\mathbf{A}^{H}$}

The saturation-robust cost function in (5) can be minimized using a variety of iterative optimization algorithms. Most of these algorithms require repeated computation of the acquisition function $\mathbf{A}$ and its adjoint $\mathbf{A}^{H}$. Efficient implementation of these functions is especially important in SAR reconstruction because of the high dimensionality of the images.

To achieve efficiency, we base our implementation on the CSA discussed in Section 2.2. Given that the CSA is an approximation to the pseudoinverse $\mathbf{A}^{\dagger}$, the acquisition function $\mathbf{A}$ has a block diagram, shown in Fig. 2, that is essentially the reverse of the one for the CSA. To implement the adjoint, we exploit the fact that the adjoint of a composition of functions is the composition of the individual adjoints in reverse order. The adjoint of the FFT is the IFFT (up to 
a scale factor) and the adjoint of multiplication by a function is multiplication by the complex conjugate of the same function. Hence the adjoint has the same block diagram as the CSA with the azimuth compression function replaced by $H_{a}^{*}\left(R_{0}, f_{\eta}\right)$ and the range compression function replaced by $P_{2 d f}^{*}\left(f_{\tau}, f_{\eta}\right)$.

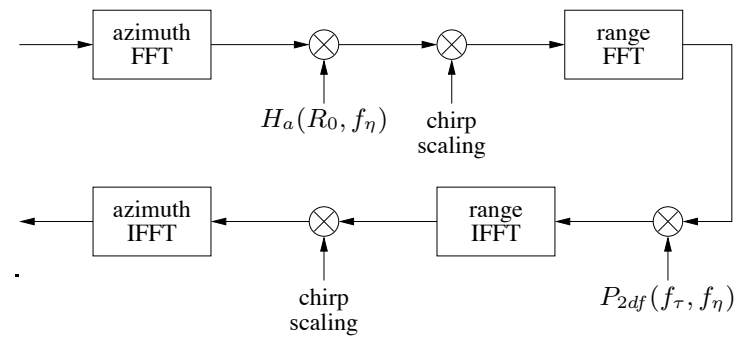

Fig. 2. Block diagram for the acquisition function $\mathbf{A}$.

We now describe our implementation of the function $\mathbf{A}$ in greater detail. We assume that the image $\mathbf{x}$ consists of a rectangular grid of point targets and we focus on the response due to a single point target of unit amplitude. Since $\mathbf{A}$ is shift-invariant in azimuth, we may equivalently specify the response in the range-Doppler (range time $\tau$, azimuth frequency $f_{\eta}$ ) domain. This is reflected in the implementation of Fig. 2 by the azimuth FFT and IFFT at the beginning and end of the block diagram. For a unit point target at range of closest approach $R_{0}$, the range-Doppler response is given approximately by

$$
H_{r d}\left(\tau, f_{\eta}\right)=H_{a}\left(R_{0}, f_{\eta}\right) p\left(\tau-\frac{2 R_{0}}{c D\left(f_{\eta}\right)}, f_{\eta}\right),
$$

where $H_{a}\left(R_{0}, f_{\eta}\right)$ is the azimuth frequency response, $p\left(\tau, f_{\eta}\right)$ is the transmitted pulse, $D\left(f_{\eta}\right)$ is the range migration factor, and $c$ is the speed of light. The pulse $p\left(\tau, f_{\eta}\right)$ is assumed to be a linear chirp and is a weak function of $f_{\eta}$ in the range-Doppler domain. A detailed derivation of (7) and formulas for the quantities appearing in (7) and Fig. 2 can be found in [2]. The response in (7) is not shiftinvariant in range because of range migration represented by $D\left(f_{\eta}\right)$. However, in the case of a linear chirp pulse, chirp scaling can be used to approximate the range response in (7) by means of a convolution with a modified chirp preceded and followed by multiplications with chirp scaling functions. The convolution is implemented using the FFT as a multiplication with $P_{2 d f}\left(f_{\tau}, f_{\eta}\right)$, the Fourier transform of $p\left(\tau, f_{\eta}\right)$ with respect to $\tau$, as shown in Fig. 2 .

Comparison of Figs. 1 and 2 shows that the complexity of computing $\mathbf{A}$ is the same as that of the CSA. All of the functions in Fig. 2 that are multiplied with the signal can be pre-computed and stored for faster computation. In a discrete-time implementation, care should be taken to use sufficiently high sampling rates to represent $H_{a}\left(R_{0}, f_{\eta}\right)$ and $P_{2 d f}\left(f_{\tau}, f_{\eta}\right)$ with minimal aliasing.

\subsection{Basic Optimization Algorithm}

We use a first-order gradient descent algorithm to minimize (5). More sophisticated second-order algorithms may be considered in future work. In each iteration, a step of size $\tau$ is taken in the direction opposite to the gradient $\mathbf{g}$, i.e., the iterates are updated as

$$
\mathbf{x} \leftarrow \mathbf{x}-\tau \mathbf{g} .
$$

The gradient $\mathbf{g}$ corresponding to (5) is given by

$$
\mathbf{g}=\mathbf{A}^{H} \mathbf{r}
$$

where $\mathbf{r}$ is the residual vector with components given in (6) and $\mathbf{A}$ and $\mathbf{A}^{H}$ are computed as described in Section 3.1. The step size $\tau$ is chosen optimally through an exact line search, which minimizes the function $f(\mathbf{y}, \mathbf{A x})$ along a line parallel to the negative gradient $-\mathbf{g}$. This leads to a 1-D convex piecewise-quadratic function that can be minimized efficiently; we omit the details from this paper.

\subsection{Azimuth Ambiguity and Acquisition Model Mismatch}

Ambiguity in the azimuth frequency domain due to undersampling is an issue affecting many SAR image formation algorithms. The azimuth sampling rate, which is equal to the pulse repetition frequency, is typically insufficient to accommodate the bandwidth of the azimuth response $H_{a}\left(R_{0}, f_{\eta}\right)$ and cannot be increased without decreasing the width of the imaged region. The undersampling results in aliasing, which manifests itself in the form of ghost images superimposed at specific azimuth and range offsets [7]. Recovery from aliasing is difficult without additional information regarding the image. It cannot be assumed, for example, that the image itself is bandlimited.

In the basic algorithm described in Sections 3.1 and 3.2, mismatch between the true acquisition system and the model used in the reconstruction poses an additional challenge. For example, the azimuth response $H_{a}\left(R_{0}, f_{\eta}\right)$ depends on parameters such as the length and weighting pattern of the antenna. The values of these parameters may not be known precisely, potentially leading to incorrect results. In particular, our experience suggests that model mismatch tends to exaggerate the ghost images caused by azimuth ambiguity.

One method to reduce ghosting is to filter the data, either nonadaptively [7] or adaptively in space [8], to remove azimuth frequencies that are most affected by aliasing. However, this approach may reduce the amount of data available for reconstruction and consequently the resolution of the resulting image.

An alternative approach that we have explored at a preliminary level is to perform ghost removal at the end of each iteration in the basic algorithm above. We exploit the ability to predict the locations of ghosts relative to the corresponding true target based on knowledge of SAR parameters. In the first iteration of the algorithm, image regions that are likely to be dominant sources of ghosts are identified as proposed in [8], first by forming a low-resolution, ambiguity-free image and then comparing the local power between regions separated by distances characteristic of ghosts. In subsequent iterations, ghosts due to dominant sources are estimated by correlating the current image with itself, one azimuth line at a time, and then subtracting the estimates from the image. Restricting the estimation and subtraction to dominant sources allows for efficient removal of ghosts.

In the context of inverse problems, our method can be thought of as adding an explicit constraint to the reconstruction optimization problem (2), specifically on the autocorrelation of the image at certain locations and shifts. This is based on the premise that large autocorrelation values should not occur in ghost-free images. An alternative would be to incorporate such knowledge in the signal model term $g(\mathbf{x})$.

\subsection{Signal Models}

Significant improvements in reconstruction accuracy can be achieved by incorporating signal models in the reconstruction. Natural images, including SAR images, exhibit predictable structure that can be exploited. In addition to the ghost reduction model described in Section 3.3 , in this work we also impose a sparsity requirement on the wavelet transform of the magnitude of the SAR image. We use 

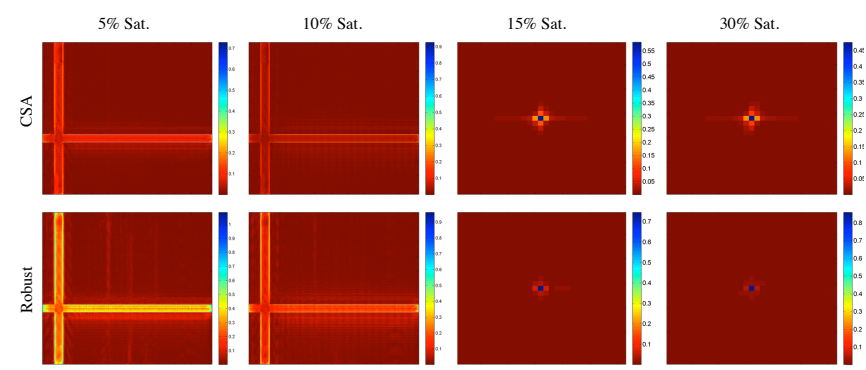

Fig. 3. Images reconstructed from saturated synthetic data.

soft thresholding in a manner similar to the FPC algorithm [9] to impose the model. At the end of every iteration of our algorithm, we transform the magnitude of the image, perform soft-thresholding, and transform back while maintaining the same phase.

The motivation for this model is that the magnitude of a SAR image looks similar to a natural image and is therefore compressible using a wavelet transform. On the other hand, the phase of the image-which depends on the variation in height of the features being imaged and undergoes severe wrapping-does not appear to offer structure that can be exploited in the reconstruction.

\section{EXPERIMENTAL RESULTS}

To better understand the behavior of our algorithms, we perform experiments using data synthesized from simple test images with an SNR of $0 \mathrm{~dB}$. In experiments with synthetic data, we do not assume a signal model or perform ghost image reduction because the examples we consider do not exhibit significant ghosting. Fig. 3 shows reconstruction results for strip and point targets with 5\%,10\%, 15\%, and $30 \%$ of the data values saturated. The top row shows images reconstructed using the CSA with no special treatment for saturated values. The bottom row shows images reconstructed using the robust consistent reconstruction algorithm described in this paper. Due to lack of space we show only a small set of the reconstructed images; similar results are obtained for different saturation levels and images.

The results in Fig. 3 demonstrate substantial improvements due to consistent reconstruction. For strip targets, the CSA results in considerable attenuation whereas consistent reconstruction produces brighter and sharper images. Similarly for point targets, the CSA yields attenuated targets and enlarged side-lobes. These distortions are significantly reduced using consistent reconstruction. Inspection of the reconstructed image values confirms these findings.

We also compare our consistent reconstruction algorithm to the CSA using satellite SAR data taken from [2], where our algorithm now includes ghost reduction and wavelet thresholding with only $30 \%$ of wavelet coefficients permitted to be non-zero. Fig. 4(a) shows an image produced by the CSA from unsaturated data, while Figs. 4(b) and 4(c) show the same image produced from a simulated $30 \%$ saturation rate using the CSA and the consistent reconstruction algorithm respectively. All of the images are scaled and displayed in a consistent way, thus demonstrating that the landmass reconstructed by the CSA under saturation in Fig. 4(b) is much fainter than in the other two images. The loss of contrast agrees with our results on synthetic images in Fig. 3. On the other hand, the consistent reconstruction in Fig. 4(c), while somewhat blurred compared to the unsaturated case, retains much of the contrast. Although not apparent at the scale used in this paper because of space constraints, the CSA images, both with and without saturation, are noisier than the image (a) CSA unsaturated

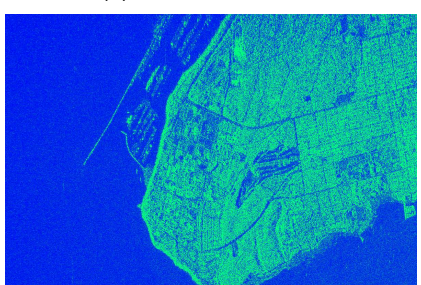

(c) Robust $30 \%$ sat

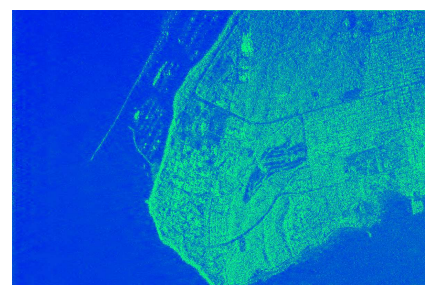

Fig. 4. Images formed from satellite SAR data using the CSA with (a) unsaturated and (b) $30 \%$ saturated data, and (c) robust consistent reconstruction with $30 \%$ saturated data.

produced by our algorithm. Further experimentation demonstrated that the improved image quality is mostly due to the saturationrobust formulation of our algorithm, while the denoising effect is mostly due to the signal model.

\section{REFERENCES}

[1] H. Runge and R. Bamler, "A novel high precision SAR focussing algorithm based on chirp scaling," in International Geoscience and Remote Sensing Symposium (IGARSS), May 1992, pp. 372-375.

[2] I. G. Cumming and F. H. Wong, Digital Processing of Synthetic Aperture Radar Data, Artech House, Jan. 2005.

[3] J. Laska, P. Boufounos, and R. Baraniuk, "Finite-range scalar quantization for compressive sensing," in Proc. Sampling Theory and Applications (SampTA), Marseille, France, May 2009.

[4] J. Laska, P. Boufounos, M. Davenport, and R. Baraniuk, "Democracy in action: Quantization, saturation, and compressive sensing," Preprint, 2009.

[5] N.T. Thao and M. Vetterli, "Reduction of the MSE in R-times oversampled A/D conversion $O(1 / R)$ to $O\left(1 / R^{2}\right)$," Signal Processing, IEEE Transactions on, vol. 42, no. 1, pp. 200-203, Jan 1994.

[6] V. K. Goyal, M. Vetterli, and N. T. Thao, "Quantized overcomplete expansions in $\mathbb{R}^{N}$ : Analysis, synthesis, and algorithms," IEEE Trans. Info. Theory, vol. 44, no. 1, pp. 16-31, Jan. 1998.

[7] F. K. Li and W. T. K. Johnson, "Ambiguities in spaceborne synthetic aperture radar systems," IEEE Trans. Aerosp. Electron. Syst., vol. 19, no. 3, pp. 389-397, May 1983.

[8] A. M. Guarnieri, "Adaptive removal of azimuth ambiguities in SAR images," IEEE Trans. Geosci. Remote Sens., vol. 43, no. 3, pp. 625-633, Mar. 2005.

[9] E. T. Hale, W. Yin, and Y. Zhang, "Fixed-point continuation for $\ell_{1}$-minimization: methodology and convergence," SIAM J. Optim., vol. 19, no. 3, pp. 1107-1130, Oct. 2008. 